Revue musicale OICRM

revue musicale oicrm

\title{
iReal Pro. Un outil technologique utilisé à des fins didactiques pour soutenir les recommandations de la recherche scientifique dans l'enseignement instrumental
}

\section{Thomas Rieppi}

Volume 4, numéro 1, 2017

URI : https://id.erudit.org/iderudit/1040303ar

DOI : https://doi.org/10.7202/1040303ar

Aller au sommaire du numéro

Éditeur(s)

Observatoire interdisciplinaire de création et recherche en musique (OICRM)

ISSN

2368-7061 (numérique)

Découvrir la revue

Citer cet article

Rieppi, T. (2017). iReal Pro. Un outil technologique utilisé à des fins didactiques pour soutenir les recommandations de la recherche scientifique dans l'enseignement instrumental. Revue musicale OICRM, 4(1), 150-158. https://doi.org/10.7202/1040303ar
Résumé de l'article

Cet article propose des façons concrètes d'intégrer à l'enseignement d'instruments de musique des recommandations issues de la recherche scientifique sur l'utilisation des technologies. En fait, il guide les praticiens sur l'utilisation du logiciel iReal Pro à l'aide d'exemples concrets modelés à partir de cours de percussions. Pour terminer, je reviens autour d'observations pédagogiques quant à l'intégration des technologies dans mes leçons de percussion et sur la manière dont leur utilisation a pu remédier, en partie, au décalage mis en avant dans la recherche scientifique. 


\title{
iReal Pro. Un outil technologique utilisé à des fins didactiques pour soutenir les recommandations de la recherche scientifique dans l'enseignement instrumental
}

\author{
Thomas Rieppi
}

\section{Résumé}

Cet article propose des façons concrètes d'intégrer à l'enseignement d'instruments de musique des recommandations issues de la recherche scientifique sur l'utilisation des technologies. En fait, il guide les praticiens sur l'utilisation du logiciel iReal Pro à l'aide d'exemples concrets modelés à partir de cours de percussions. Pour terminer, je reviens autour d'observations pédagogiques quant à l'intégration des technologies dans mes leçons de percussion et sur la manière dont leur utilisation a pu remédier, en partie, au décalage mis en avant dans la recherche scientifique.

Mots clés : créativité ; éducation musicale extrascolaire ; logiciels ; technologies ; travail instrumental.

\section{Abstract}

This article proposes concrete ways to integrate recommendations from scientific research concerning the usage of technologies in music teaching. In fact, it guides practitioners on how to use the iReal Pro software using examples modeled from percussion courses. Finally, I made some pedagogical observations concerning the integration of technologies in my percussion lessons and how iReal Pro provides some opportunities to fill the gap demonstrated in the scientific literature.

Keywords: creativity; instrumental practice; extracurricular music eduction; software; technologies. 


\section{PRÉSENTATION DU SUJET}

Depuis plusieurs années, les technologies ne cessent de se développer et sont utilisées au quotidien par les différentes couches de la société, notamment dans le domaine artistique et plus particulièrement pour l'apprentissage et l'enseignement de la musique auprès des jeunes. En effet, l'émergence des technologies numériques a radicalement changé les pratiques musicales des jeunes depuis les 20 dernières années (Kress 2010). La chercheuse Susan O'Neill (2013) souligne d'ailleurs l'importance pour les enseignants en musique de s'adapter à ces nouvelles réalités afin que leur pédagogie soit plus en phase avec les goûts musicaux des jeunes et qu'elle s'inscrive davantage dans le courant actuel des technologies. En fait, l'intégration des technologies à leurs pratiques pédagogiques permettrait d'établir des contextes d'apprentissage plus significatifs pour le jeune, car les expériences musicales vécues seraient plus près du monde dans lequel il vit. En revanche, l'adaptation de leur enseignement à ces nouvelles réalités numériques représente un défi de taille, car le jeune possède souvent plus de connaissances que son professeur sur le plan des technologies. D'ailleurs, les élèves sont communément considérés comme des "digital natives " : ils maîtrisent les technologies comme une langue maternelle alors que pour les enseignants, qualifiés de "digital immigrants", celles-ci constituent, dans une certaine mesure, l'équivalent d'une langue seconde (Prensky 2001a, 2001b). Ce décalage exige de la part des pédagogues de rattraper leur retard sur le plan technologique pour être en mesure de mieux exploiter cette dimension dans leur enseignement et pour mieux guider l'apprentissage des élèves à l'aide des technologies.

On retrouve aujourd'hui plusieurs logiciels et applications mobiles consacrés à la musique qui peuvent aussi être facilement utilisés pour apprendre la musique et intégrés aux cours d'instruments, dont le logiciel $\underline{i R e a l ~ P r o}$. Ce texte vise ainsi à présenter ce logiciel et à proposer des manières de l'intégrer aux cours d'instruments en musique classique. De façon plus précise, je présenterai les différents avantages liés à son utilisation pour diversifier la formation instrumentale des musiciens classiques et j'en suggérerai des applications à l'aide d'exemples concrets d'utilisation modelés pour les cours de percussions à partir d'expériences réalisées avec mes anciens élèves de tutorat du Conservatoire de Toulouse et de la participation d'un ami percussionniste. Pour terminer, je reviendrai sur quelques observations pédagogiques quant à l'intégration des technologies dans mes leçons de percussion et sur la manière dont leur utilisation a pu remédier, en partie, au décalage mis en avant dans la recherche scientifique.

\section{PréSentation D'iReal Pro}

iReal Pro est un logiciel qui existe également sous forme d'application mobile qui permet de jouer des morceaux de musique accompagnés par un groupe de musiciens virtuels. En un clic, il est notamment possible de modifier le tempo, la tonalité, le style et les balances de l'œuvre jouée. Il permet aussi de créer, d'enregistrer, d'envoyer et d'imprimer des grilles d'accords. On peut y apprendre de nouveaux accords grâce à un outil intégré au système et visualiser la construction de ceux-ci sur une portée 
ainsi que leurs différents doigtés pour la guitare, le piano et le ukulélé. Enfin, cette application offre régulièrement des mises à jour ajoutant de nouvelles possibilités d'utilisation.

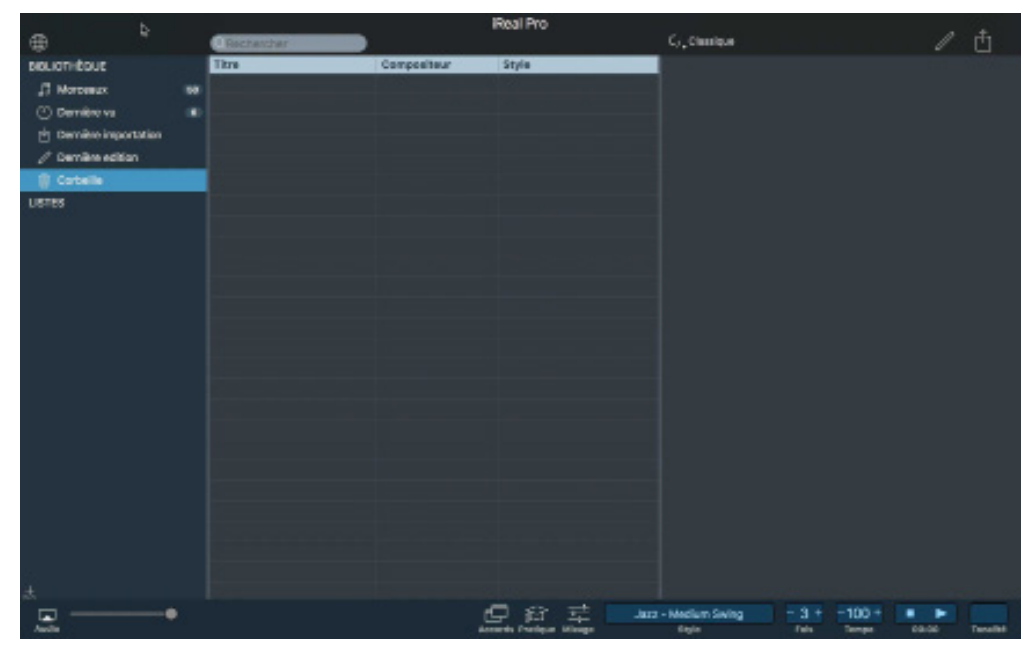

Extrait vidéo 1 : Tutoriel de présentation d'iReal Pro.

\section{RÉFLEXIONS SUR SON UTILISATION}

\section{Réflexion personnelle}

En entrant au Conservatoire, j'ai développé un intérêt pour les musiques improvisées. Pour acquérir des compétences dans ce domaine, j'ai suivi des cours de vibraphone jazz en dehors du Conservatoire basés sur des activités d'improvisation. Le professeur utilisait le logiciel iReal Pro qui, à l'époque, s'intitulait IRealb, pour guider l'apprentissage de musiques improvisées. Les possibilités offertes par cette application m'ont de suite séduit et m'ont permis de développer mes habiletés d'improvisateur. $\underline{\text { Real Pro }}$ m'a aussi permis de découvrir différents styles musicaux, d'aborder l'improvisation de façon structurée et d'avoir un outil de travail non négligeable dans ma pratique personnelle tout en construisant ces nouvelles habiletés de façon autonome.

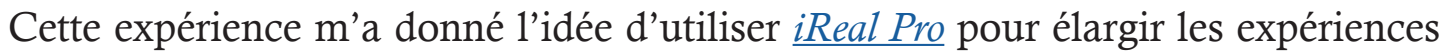
musicales de mes élèves de percussion, qui suivent un parcours similaire au mien. Il s'agit, en effet, d'étudiants apprenant la musique sous une approche traditionnelle de type "Conservatoire ", que j'initie à l'improvisation, à divers styles musicaux, ou que je guide pour acquérir des savoirs et savoir-faire musicaux de façon plus créative.

\section{EXEMPLES D'UTILISATION}

Les différentes fonctions d'iReal Pro permettent l'apprentissage de la musique à l'aide d'une approche innovante tout en développant une certaine autonomie pour le travail individuel et quotidien effectué à la maison. De plus, il s'agit d'un outil technologique ayant le potentiel d'influencer positivement la motivation de l'élève dans ses séances de répétitions. La section suivante propose quelques exemples d'utilisation de ce logiciel, même si les possibilités sont beaucoup plus vastes que celles illustrées ici. 


\section{La découverte des styles}

Grâce au logiciel, les élèves peuvent découvrir et apprendre à jouer de nombreux styles de musique qui se divisent en trois grandes catégories : jazz, latin et pop. L'acquisition du logiciel donne également accès à des forums où l'on peut télécharger d'autres pièces s'inscrivant dans d'autres styles musicaux (classique, brésilien, fusion, rock, etc.) et pouvant être jouées dans un style jazz, latin ou pop.

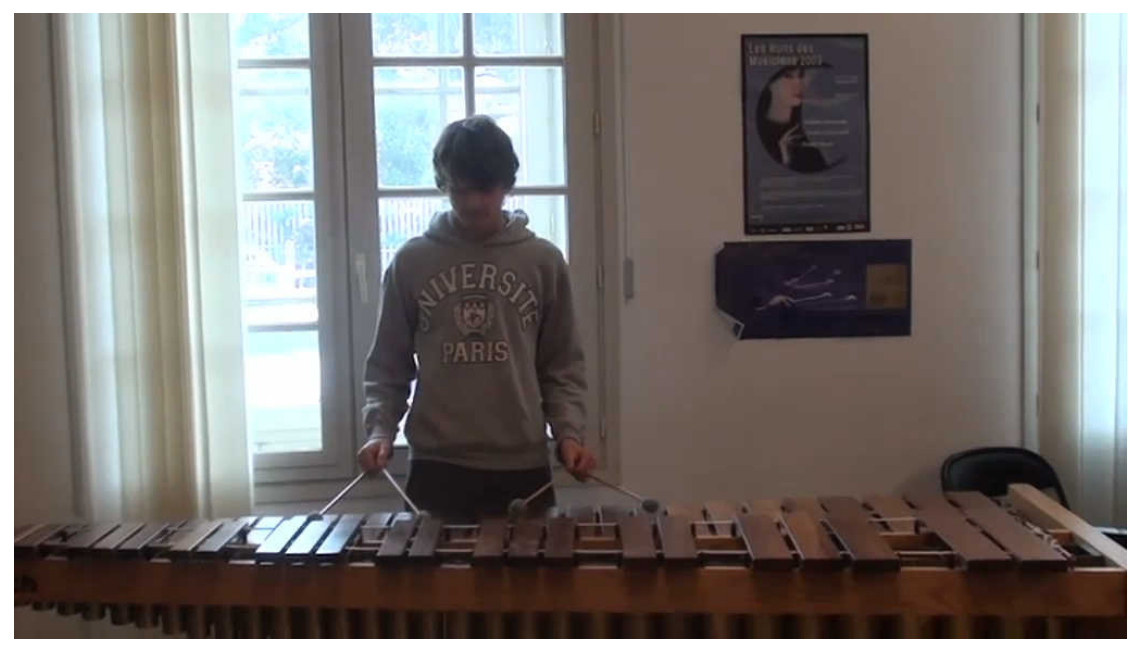

Extrait vidéo 2 : Exercices techniques à travers le "Canon de Pachelbel».

Dans cet extrait, l'élève répète des exercices techniques au marimba à l'aide d'une grille d'accords tirés du «Canon de Pachelbel », laquelle est intégrée dans iReal Pro. Cette approche lui permet de répéter la technique instrumentale en apprentissage sur un accompagnement " pop ", tout en utilisant une pièce de musique classique. De cette manière, l'élève travaille un savoir "technique " à l'aide d'une approche expressive qui s'avère plus stimulante pour lui.

\section{L'interprétation et la composition}

iReal Pro permet de composer des grilles d'accords de façon très simple. Cette caractéristique du logiciel facilite la création de liens entre les cours d'analyse, de formation auditive, d'écriture et d'instrument par le biais de la composition.

Plus concrètement, l'élève peut d'abord analyser harmoniquement une de ses pièces musicales classiques pour en identifier les accords. Il peut ensuite entrer les accords dans le logiciel sous le format d'une grille. Cette opération lui permet de travailler sa pièce avec un accompagnement de style jazz, latin ou pop qu'il aura choisi. Cette approche créative du travail peut lui inspirer différentes pistes d'interprétation de sa pièce.

L'élève peut également composer différentes grilles d'accord à partir desquelles il peut composer une pièce originale ou improviser. De plus, l'utilisation d' $\underline{\text { Real Pro }}$ pour composer ses propres œuvres peut aussi l'aider à mieux maîtriser différents savoirs musicaux théoriques. 


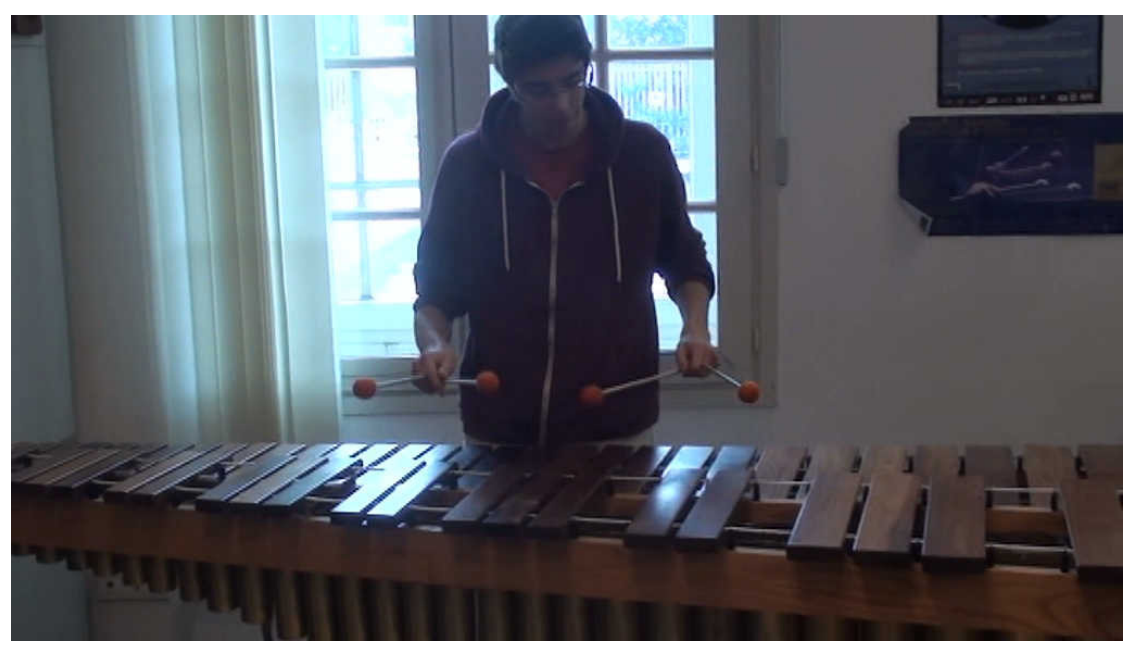

Extrait vidéo 3 : Travail du répertoire "Rotation 1, d'Eric Sammut ».

Cet exemple illustre l'utilisation du logiciel pour travailler une pièce solo du répertoire de percussions basée sur une analyse harmonique de l'œuvre. Ici, l'élève a analysé un passage de la pièce, puis a transcrit la grille d'accord dans le logiciel en vue de répéter ce même passage et d'inspirer son travail d'interprétation. Ce travail lui permet également d'analyser et de répéter son répertoire de manière plus créative.

Vers une technique expressive

Il peut s'avérer difficile pour les élèves de se motiver à répéter des exercices techniques. En effet, l'élève perçoit souvent l'apprentissage de la technique instrumentale comme une activité ennuyante. L'utilisation d'iReal Pro peut atténuer ou contourner ce problème, car l'outil permet de refaire les exercices à l'aide d'une approche plus expressive.

À titre d'exemple, prenons un exercice technique au marimba à quatre baguettes qui consiste à travailler la technique à partir d'un mouvement des avant-bras appelé « rotations ». Quand les élèves répètent cet exercice, ils n'y trouvent pas nécessairement un intérêt direct. De plus, le fait de leur mentionner que cet exercice leur servira plus tard, dans des pièces du répertoire, n'est pas forcément un argument suffisamment fort pour les motiver à le répéter. Cependant, si l'élève peut composer sa propre grille d'accords et la réaliser à l'aide d'un accompagnement basé sur différents styles musicaux par des instruments qu'il a choisis, ou encore s'il travaille la technique à partir d'une pièce qu'il affectionne particulièrement, il peut être plus motivé à répéter cette technique instrumentale. En outre, cette approche l'aidera à développer de nombreuses compétences transversales. En effet, un travail d'analyse, de réflexion, de composition, de découverte des styles musicaux s'ajoutera à son travail technique. 


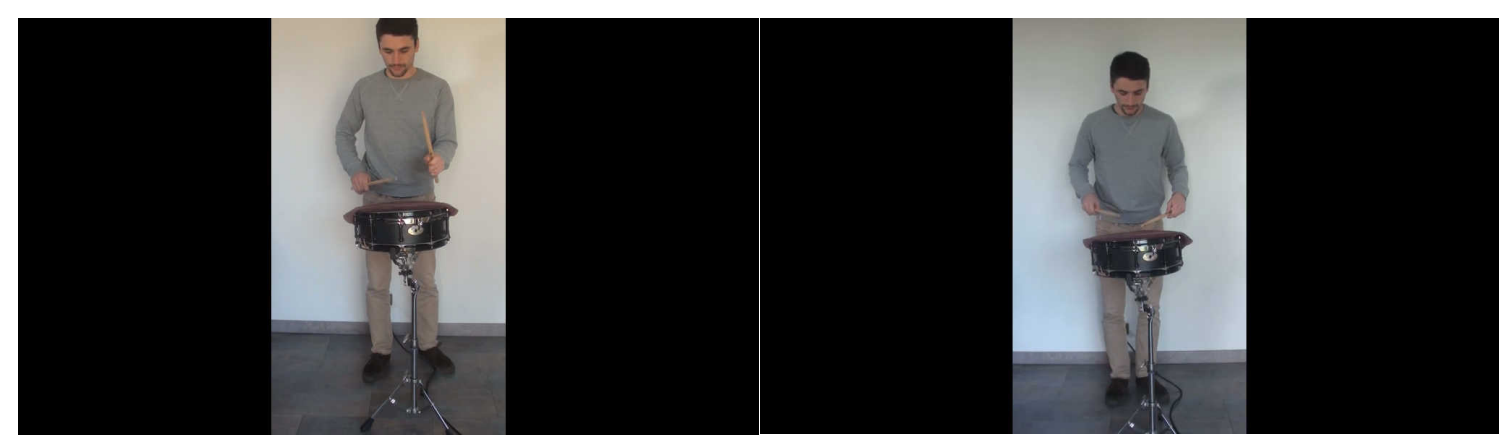

Extrait vidéo 4 : Répétition d'un rudiment de percussions appelé " moulins" sur une base de doubles croches autour d'une improvisation.
Extrait vidéo 5 : Répétition du même rudiment, mais, cette fois, sur une base de sextolets.

Dans ces extraits, Simon répète des séquences techniques accompagnées par une basse électrique et des harmonies au piano. Cette manière de répéter lui permet de développer l'écoute, la créativité autour de l'improvisation et permet une approche plus ludique du travail technique. De plus, elle permet d'éviter une monotonie liée au travail technique à l'aide du métronome qui est remplacé, ici, par un vrai accompagnement musical qu'il peut modifier à sa guise.

\section{La transposition}

Le travail de la transposition est une habileté musicale parfois négligée auprès des musiciens classiques. Pourtant, le fait de transposer une phrase peut permettre de mieux se l'approprier et développe plus rapidement le jeu à l'oreille. Avec le logiciel, en un clic, il est possible de transposer une grille et de se servir d'un thème très simple pour les plus jeunes afin de l'obtenir dans toutes les tonalités.

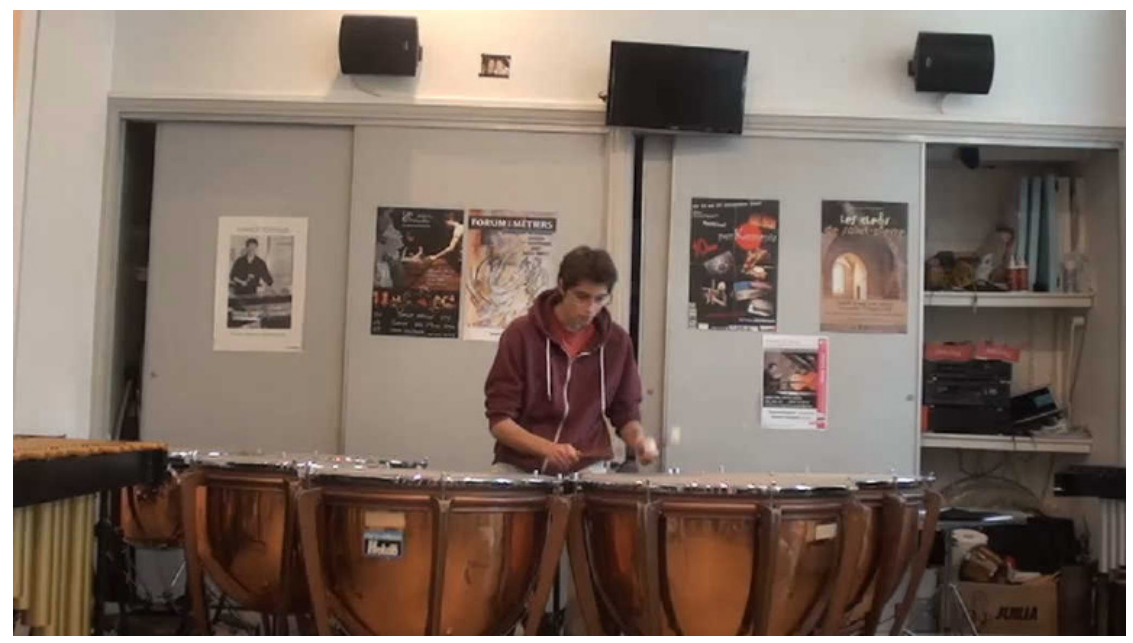

Extrait vidéo 6: Blues en do aux timbales.

Dans cet extrait, Rémy travaille le son aux timbales sur un blues en $d o$. En premier lieu, cet exercice lui permet de travailler le pédalage, c'est-à-dire les changements de notes des timbales en utilisant les pédales. En effet, le travail du pédalage aux timbales est très difficile et particulièrement important afin de jouer juste. 
Ainsi, Rémy va développer le jeu des pédales afin d'être le plus juste possible pour jouer avec les harmonies de l'accompagnement, tout en découvrant de nouveaux styles musicaux. En l'occurrence, dans cet extrait, il s'agit d'un blues qui ne fait pas partie du répertoire habituel aux timbales. En second lieu, il est important de préciser que l'application facilite grandement le travail de transposition. À cet effet, il suffit d'un clic pour modifier la tonalité de l'accompagnement, ce qui s'avère très utile pour le travail des timbales.

\section{L'improvisation}

iReal Pro s'avère un outil privilégié pour travailler l'improvisation avec des pièces allant des Beatles à Miles Davis en passant par des mélodies traditionnelles provenant de différents pays et cultures. En fait, ce travail d'improvisation peut s'effectuer à partir de différentes pièces qui sont à portée de main. De plus, les élèves ont aussi la possibilité d'improviser sur des grilles d'accords qu'ils ont eux-mêmes composées en sélectionnant l'outil " nouveau morceau ». Dans un autre ordre d'idées, les activités d'improvisations via iReal Pro permettent de guider les élèves dans leur travail personnel. Ainsi, une section " bibliothèque d'accords » donne la possibilité de voir comment sont conçus les différents accords et gammes tout en donnant des possibilités de doigtés pour les jouer à la guitare, au piano ou au ukulélé.

Dans ces différents extraits, Rémy et Luc effectuent une improvisation à l'aide d'un accompagnement qu'ils ont eux-mêmes composé à partir d'une grille d'accords. Cette manière de répéter leur permet de jouer en groupe, de développer leur habileté à écouter, leur créativité autour de la composition et de l'improvisation, et de s'immerger dans différents styles musicaux.

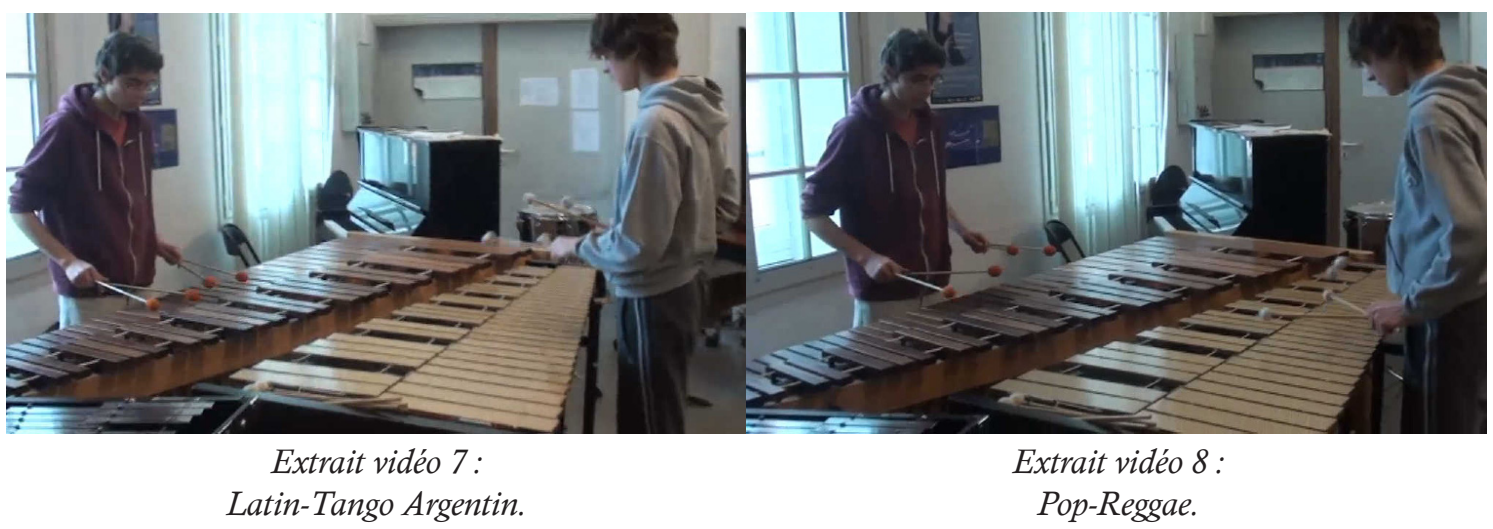




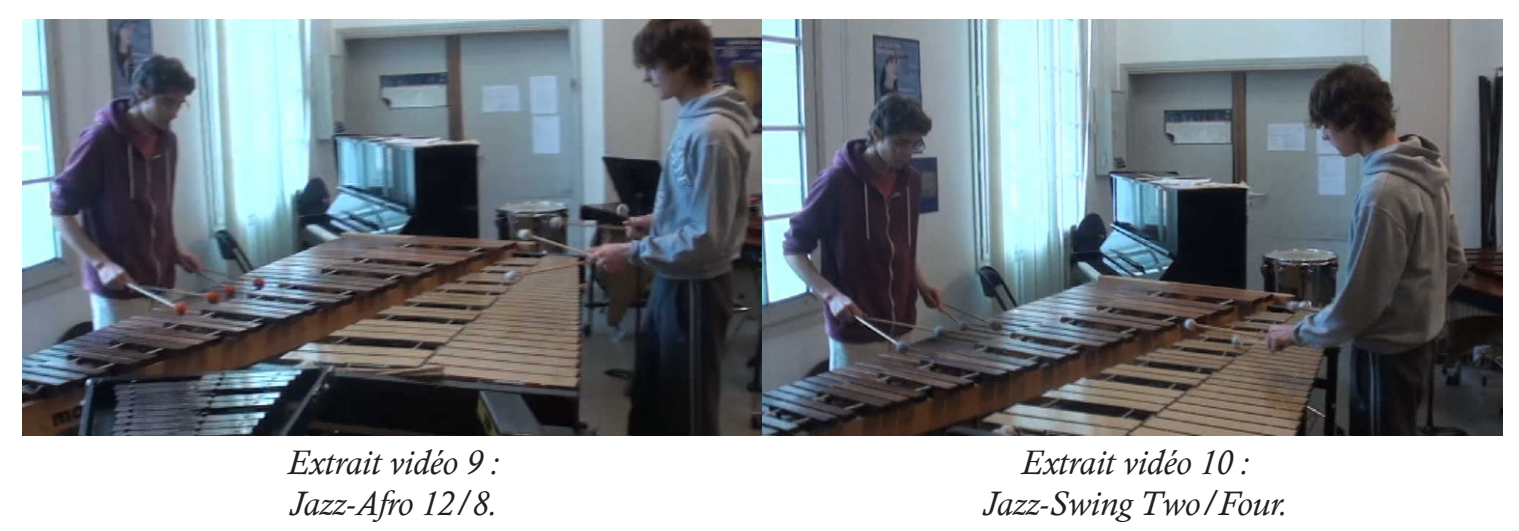

Ces divers extraits sont tirés de véritables leçons. Il est intéressant de constater dans la séquence pop-reggae que Rémy, à gauche de l'écran, joue d'abord l'accompagnement sur les temps. Par le biais de l'écoute, il prend ensuite conscience que ce style musical requiert un appui de l'accompagnement sur le contretemps.

\section{OBSERVATIONS PÉDAGOGIQUES}

Durant les leçons de percussion que je donne, j'ai pu observer que les plus jeunes élèves étaient peu motivés à répéter avec un métronome pendant leurs séances de répétition à la maison. En effet, l'utilisation du métronome est souvent mal comprise et perçue de manière négative par les jeunes. Cependant, en ayant recours à iReal Pro, j'ai pu voir en peu de temps des changements significatifs dans l'utilisation du métronome. De fait, l'application permet de remplacer le métronome par divers accompagnements de percussions et de rendre son usage plus ludique pour les jeunes. De plus, j'ai observé que les élèves n'hésitent pas à chercher différents accompagnements dans l'application, ce qui les amène à prendre du plaisir à répéter avec. À titre d'exemple, un élève est venu à sa leçon en me montrant fièrement sur quels styles de musique à trois temps il pouvait pratiquer les séquences rythmiques qu'il devait répéter durant la semaine. Il avait joint à cela une petite recherche sur le Web dans le but d'expliquer à ses camarades l'origine de ces styles musicaux. Cette découverte a, sans aucun doute, développé son sentiment de compétence, et favorise sa motivation et son engagement dans la classe de percussion. De plus, comme il s'agissait de leçons de groupe, les autres élèves ont voulu, par la suite, faire de même. Ainsi, les élèves venaient chaque semaine aux leçons en expliquant les différentes possibilités de s'exercer avec le logiciel, la manière dont ils avaient répété une séquence rythmique sur un style musical, et donnaient une brève présentation du style musical en question.

Chez les plus grands, j'ai pu constater qu'iReal Pro est perçu comme un outil leur permettant de toucher à l'improvisation dans leur parcours musical et de combler un manque présent dans la formation classique, tout en ouvrant des portes sur la composition. De plus, j'ai remarqué que plusieurs de mes élèves âgés de 14 à 18 ans utilisaient l'application pour réaliser des projets musicaux en groupe en dehors de l'école. À titre d'exemple, des élèves l'ont employé pour vérifier la construction de gammes ou d'accords grâce à la section «bibliothèque d'accords ». 


\section{CONCLUSION}

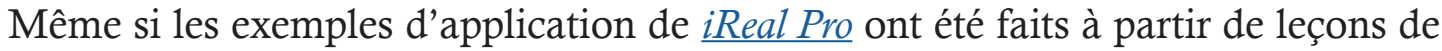
percussions, cet outil peut parfaitement convenir pour tous les instruments. Il s'agit d'un outil formidable permettant aux enseignants de se rapprocher du monde actuel et de faire appel à des technologies significatives pour les jeunes. De plus, son usage peut développer des compétences théoriques, d'interprétation et de créativité, et pallier aux manques souvent ressentis par les élèves suivant un cursus de formation musicale classique. Enfin, mon expérience liée à son utilisation m'a permis de constater qu'iReal Pro peut mobiliser positivement la motivation des étudiants envers leur apprentissage musical.

BIBLIOGRAPHIE

Kress, Gunther (2010), Multimodality. A Social Semiotic Approach to Contemporary Communication, London, Routledge.

O'Neill, Susan A. (2013), "Using Affordances and Constraints to Evaluate Young People's Transformative Music Engagement in Today's Digital Age », The Eight International Research in Music Education (RIME) Conference, Université d'Exeter, 9-13 avril.

Prensky, Marc (2001a), « Digital Natives, Digital Immigrants », On the Horizon, vol. 9, n 5 (octobre), p. 1-6, http://www.marcprensky.com/writing/Prensky\%20-\%20Digital\%20Natives, $\% 20$ Digital\%20Immigrants\%20-\%20Part1.pdf, consulté le 12 avril 2017.

Prensky, Marc (2001b), "Digital Natives, Digital Immigrants, Part II. Do They Really Think Differently?», On the Horizon, vol. 9, nº 6 (November/December), p. 1-6, http://www.marcprensky. com/writing/Prensky\%20-\%20Digital\%20Natives, \%20Digital\%20Immigrants\%20-\%20Part2.pdf, consulté le 12 avril 2017. 